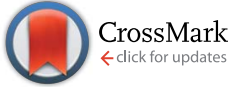

Cite this: Chem. Sci., 2015, 6, 5719

Received 10th June 2015

Accepted 3rd July 2015

DOI: $10.1039 / \mathrm{cssc02086g}$

www.rsc.org/chemicalscience

\section{Rational synthesis of normal, abnormal and anionic NHC-gallium alkyl complexes: structural, stability and isomerization insights $\dagger$}

\author{
Marina Uzelac, Alberto Hernán-Gómez, David R. Armstrong, Alan R. Kennedy \\ and Eva Hevia*
}

Advancing the rational design of main-group $\mathrm{N}$-heterocyclic carbene complexes, this study reports the synthesis, X-ray crystallographic and NMR spectroscopic characterisation of a novel series of $\mathrm{Ga}$ complexes containing neutral or anionic NHC ligands using the unsaturated carbene $\operatorname{IPr}(\operatorname{IPr}=1,3$-bis(2,6-di-isopropylphenyl)imidazol-2-ylidene). Starting from normal adduct $\mathrm{GaR}_{3} \cdot \operatorname{IPr}(1)\left(\mathrm{R}=\mathrm{CH}_{2} \mathrm{SiMe}_{3}\right)$, the addition of polar LiR led to the formation of $\mathrm{NHC}$-stabilised gallate species IPr. $\mathrm{LiGaR}_{4}(2)$, resulting from co-complexation of the single-metal species. Contrastingly, reversing the order of addition of these organometallic reagents, by treating unsaturated free $\mathrm{IPr}$, first with LiR followed by $\mathrm{GaR}_{3}$, furnished novel heteroleptic gallate (THF) ${ }_{2} \mathrm{Li}\left[: \mathrm{C}\left\{\left[\mathrm{N}\left(2,6-\mathrm{iPr}_{2} \mathrm{C}_{6} \mathrm{H}_{3}\right)\right]_{2} \mathrm{CHCGa}\left(\mathrm{CH}_{2} \mathrm{SiMe}_{3}\right)_{3}\right\}\right]$ (3), which contains an anionic NHC ligand acting as an unsymmetrical bridge between the two metals, coordinating through its abnormal C4 position to Ga and through its normal C2 position to Li. Electrophilic interception studies of 3 using methyl triflate (MeOTf), methanol and imidazolium salt (IMes. $\mathrm{HCl}$ ) led to the isolation and structural elucidation of the two novel neutral abnormal NHC (aNHC) complexes $\left[\mathrm{CH}_{3} \mathrm{C}\{[\mathrm{N}(2,6-\right.$ $\left.\left.\left.\left.\mathrm{iPr}_{2} \mathrm{C}_{6} \mathrm{H}_{3}\right)\right]_{2} \mathrm{CHCGa}\left(\mathrm{CH}_{2} \mathrm{SiMe}_{3}\right)_{3}\right\}\right]$ (4) and $\operatorname{alPr} \cdot \mathrm{GaR}_{3}$ (5) (alPr $\left.=\mathrm{HC}\left\{\left[\mathrm{N}\left(2,6-\mathrm{iPr}_{2} \mathrm{C}_{6} \mathrm{H}_{3}\right)\right]_{2} \mathrm{CHC}\right\}\right)$. These studies disclose the preference of the anionic $\operatorname{IPr}$ ligand present in 3 to react with electrophiles via its $\mathrm{C} 2$ position, leaving its $\mathrm{Ga}-\mathrm{C} 4$ bond intact. Abnormal complex 5 can also be accessed by a thermally induced rearrangement of its normal isomer 1. Combining NMR spectroscopic and kinetic studies with DFT calculations, new light has been shed on this intriguing transformation, which suggests that it occurs via a dissociative mechanism, highlighting the importance of the donor ability of the solvent used in these thermal isomerizations as well as the steric bulk of the substituents on the NHC and the Ga reagent. These findings intimate that relief of the steric hindrance around Ga by forming an abnormal complex is a key driving force behind these rearrangements.

\section{Introduction}

Over the past two decades, N-heterocyclic carbenes (NHCs), in particular imidazol-2-ylidenes, have progressed from mere curiosities to commodity neutral $\sigma$-donor ligands with a multitude of applications in synthesis and materials. ${ }^{1}$ Typically, the carbene centre is located between the two nitrogen atoms (C2 position) allowing $\pi$-donation by both adjacent $\mathrm{N}$-heteroatoms into the empty $\mathrm{p}_{\pi}$ orbital of the carbene, which makes these ligands remarkably more stable than other non-cyclic, allcarbon counterparts. ${ }^{2,3}$ Acting as strong $\sigma$-donors, these

WestCHEM, Department of Pure and Applied Chemistry, University of Strathclyde, Glasgow, G1 1XL, UK. E-mail: eva.hevia@strath.ac.uk

$\dagger$ Dedicated to the memory of Paul von R. Schleyer.

\$ Electronic supplementary information (ESI) available: CIF files giving crystallographic results, experimental details and copies of the NMR spectra. CCDC 1405459-1405464. For ESI and crystallographic data in CIF or other electronic format see DOI: $10.1039 / \mathrm{c} 5 \mathrm{sc} 02086 \mathrm{~g}$ versatile ligands have been pivotal to recent breakthroughs in transition-metal catalysis. ${ }^{\mathbf{4}-\mathbf{9}}$ Furthermore, the application of NHCs to main group chemistry has enabled the stabilisation of novel low valent compounds, ${ }^{10-17}$ as well as the development of several frustrated Lewis pair (FLP) systems. ${ }^{\mathbf{1 8 , 1 9}}$

In parallel to these studies, a different type of NHC complex has been developed where the imidazole ring binds to the metal centre through its backbone. These less stabilised carbenes, where there is only one $\mathrm{N}$-atom adjacent to their carbenic- $\mathrm{C},{ }^{20}$ have been termed as abnormal (or mesoionic) NHCs (aNHCs). ${ }^{21-23}$ Following Crabtree's seminal report in 2001 of the first transition-metal complex with an aNHC, several other examples have been prepared. ${ }^{24}$ However, it was only in 2009 when Bertrand succeeded in the isolation of the first stable free aNHC by the elegant deprotonation of a 1,2,3,4-tetraarylated imidazolium chloride. ${ }^{25,26}$

Interestingly, experimental and theoretical studies point to aNHCs being better donors than their normal counterparts, which is in part attributed to their reduced steric 
congestion..$^{25,27-29}$ Thus Layfield has recently reported a thermally induced rearrangement of $\mathrm{IPr} \cdot \mathrm{Fe}(\mathrm{HMDS})_{2}[\mathrm{IPr}=1,3$-bis(2,6-di-isopropylphenyl)imidazol-2-ylidene, HMDS = 1,1,1,3,3,3hexamethyldisilazide] which after $3 \mathrm{~h}$ in refluxing toluene evolves to its abnormal isomer. ${ }^{30}$ Within main group chemistry, the number of complexes containing aNHCs remains very limited. Thus the first example of an adduct of this type, a substituted phosphinidene complex, was reported by Carty in 2006. ${ }^{31}$ Related thermal rearrangements as that mentioned above in iron chemistry have been proposed for tris(pentafluorophenyl)borane NHC systems which exhibit FLP chemistry. $^{32-34}$ Similarly, within group 13 Dagorne has shown the isomerization of $\mathrm{I}^{t} \mathrm{Bu} \cdot \mathrm{AlMe}_{3}[\mathrm{IBu}=1,3$-bis(tert-butyl)imidazol-2ylidene] to its $\mathrm{C} 4$ bound isomer $\left(\mathrm{aI}^{t} \mathrm{Bu} \cdot \mathrm{AlMe}_{3}\right)$ at room temperature in THF, although the mechanisms involved in these processes remain unclear. ${ }^{35}$

In addition to these isomerization studies, Robinson has demonstrated that anionic NHCs, resulting from the lithiation of the imidazole backbone of unsaturated $\mathrm{NHCs}^{36}$ can be employed as platforms to access aNHC-complexes of B and $\mathrm{Zn}$ by quenching the relevant anionic $\mathrm{B}$ or $\mathrm{Zn}$ complex with a suitable electrophile such as $\mathrm{HCl} \cdot \mathrm{NEt}_{3}$ or MeOTf. ${ }^{37-39}$ More recently, Lavallo et al. have shown that implementing carborane anions as $\mathrm{N}$-imidazolium substituents allows the selective formation of both normal and abnormal NHC constitutional isomers from a single precursor. ${ }^{40}$ In addition, the authors report a thermal isomerization from abnormal to normal NHC, which can be proton catalyzed.

Building on these precedents, exporting this chemistry to the heavier group 13 metal gallium, and using the unsaturated carbene IPr as a case study, here we report a novel series of normal, anionic and abnormal NHC complexes derived from the same metal fragment, tris(alkyl)gallium $\mathrm{Ga}\left(\mathrm{CH}_{2} \mathrm{SiMe}_{3}\right)_{3}$. Combining X-ray crystallographic, kinetic and spectroscopic studies with theoretical investigations we assess the constitution and stability of these complexes. An insightful comparison between two alternative synthetic methods to access aNHC complexes: namely metallation/electrophilic interception and thermal isomerisation, is also provided. It should be noted that although there are several examples of $\mathrm{Ga}-\mathrm{NHC}$ adducts reported in the literature, ${ }^{\mathbf{4 1 - 4 5}}$ with some of them finding applications as $\pi$-acid catalysts, ${ }^{46,47}$ the only abnormal NHC complex known to date aIPr $\cdot \mathrm{GaCl}_{3}{ }^{39}$ was reported as recently as 2014, although its synthesis is not straightforward as it was obtained by transmetallation of $\mathrm{GaCl}_{3}$ with an anionic NHC mixed Li-B complex.

\section{Results and discussion}

\section{NHC-stabilised lithium gallate complexes}

We started our studies by reacting equimolar amounts of IPr and trimethylsilylmethylgallium(III) ${ }^{48}\left(\mathrm{GaR}_{3}\right)\left[\mathrm{R}=\mathrm{CH}_{2} \mathrm{SiMe}_{3}\right]$ at room temperature in non-polar hexane which afforded colourless crystals of the adduct $\mathrm{IPr} \cdot \mathrm{GaR}_{3}(\mathbf{1})$ in a $75 \%$ isolated yield (Scheme 1). The molecular structure of $\mathbf{1}$ (Fig. 1) was elucidated by a single crystal X-ray diffraction analysis which revealed the formation of a complex with the four-carbon-coordinated gallium atom attached to three alkyl groups and the $\mathrm{C} 2$ (i.e. $\mathrm{C} 1$ in Fig. 1) of a neutral carbene. A distorted tetrahedral geometry adopted by $\mathrm{Ga}$ centre is evidenced by the $\mathrm{C}-\mathrm{Ga}-\mathrm{C}$ bond angles which range from $96.36(6)^{\circ}$ to $119.43(7)^{\circ}$ (average angle $\left.108.79^{\circ}\right)$. The $\mathrm{Ga}-\mathrm{C}_{\text {alkyl }}$ distances range from $2.0034(15) \AA$ to 2.0164(16) $\AA$ (mean $2.0106 \AA$ ) which is only slightly elongated (by $\sim 2.5 \%$ ) when compared to parent monomeric $\mathrm{GaR}_{3}(\mathrm{Ga}-\mathrm{C}$

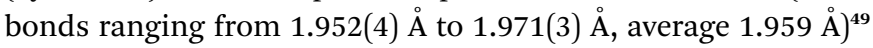
in agreement with the increase in the coordination number of Ga in 1.

Noticeably, the Ga- $\mathrm{C}_{\mathrm{NHC}}$ distance of $2.1960(16) \AA$ is significantly longer compared to that observed in the related Ga halide complex $\mathrm{IPr} \cdot \mathrm{GaCl}_{3}(2.016(2) \AA) .{ }^{42}$ This elongation can be rationalised in terms of a combination of the greater steric congestion in $\mathbf{1}$ imposed by the monosilyl groups as well as the stronger Lewis acidity of $\mathrm{GaCl}_{3}$ compared to $\mathrm{GaR}_{3}$.

Despite the long $\mathrm{Ga}-\mathrm{C} 1$ distance, it should be noted that 1 retains its integrity in $\mathrm{C}_{6} \mathrm{D}_{6}$ solution as evidenced by DOSY NMR studies, which show that the IPr and monosilyl groups belong to the same sized species, as the cross-point for both ligand resonances are aligned in the second dimension (average $D$ value $=6.2 \times 10^{-10} \mathrm{~m}^{2} \mathrm{~s}^{-1}$; see Fig. $\mathrm{S} 39$ in ESI $\$$ ). The most informative resonance in the ${ }^{13} \mathrm{C}$ NMR spectrum is that for the carbenic carbon at $186.6 \mathrm{ppm}$, a chemical shift consistent with retention of the $\mathrm{Ga}-\mathrm{C}$ bond in solution. ${ }^{50}$

Next the reactivity of 1 towards $\mathrm{LiCH}_{2} \mathrm{SiMe}_{3}$ was investigated. Previous work by Roesky and Stalke ${ }^{51}$ has shown that when borane adduct $\mathrm{IPr} \cdot \mathrm{BH}_{3}$ is treated with $\mathrm{BuLi}$, lithiation of the $\mathrm{C} 4$ position of the imidazole ring takes place affording an anionic $\mathrm{NHC}$ which binds through its $\mathrm{C} 4$ position to $\mathrm{Li}$, leaving the $\mathrm{B}-\mathrm{C} 2$ bond untouched. ${ }^{51}$ Similar reactivity has also been described for the alkylborane $\mathrm{IPr} \cdot \mathrm{BEt}_{3} \cdot{ }^{36}$

Interestingly in our studies, the polar organometallic RLi fails to deprotonate the NHC ligand of 1, affording instead lithium gallate $\left[\mathrm{IPr} \cdot \mathrm{LiGa}\left(\mathrm{CH}_{2} \mathrm{SiMe}_{3}\right)_{4}\right](2)$ in an isolated yield of $48 \%$. Single crystal X-ray diffraction analysis established the molecular structure of $\left[\mathrm{IPr} \cdot \mathrm{LiGa}\left(\mathrm{CH}_{2} \mathrm{SiMe}_{3}\right)_{4}\right]$ which represents to the best of our knowledge the first example of an alkali-metal gallate stabilised by an NHC ligand (Fig. 2). ${ }^{52}$ Compound 2 exhibits a contacted ion pair (CIP) motif where the two metals

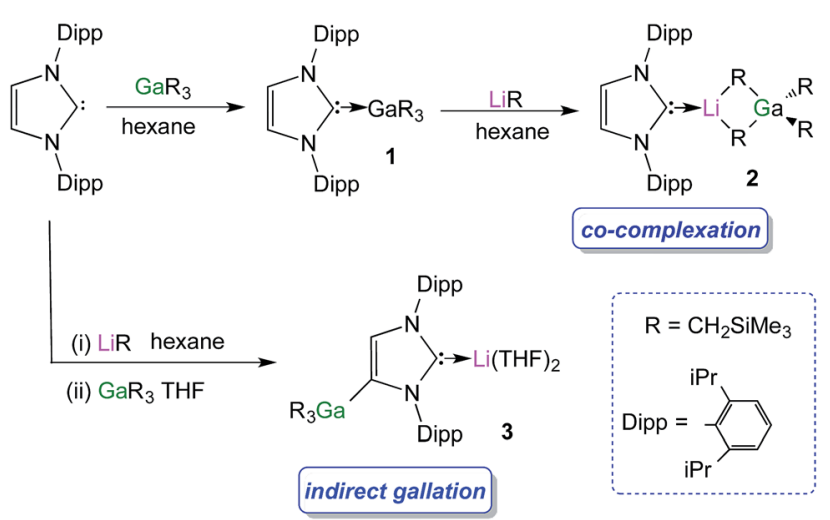

Scheme 1 Synthesis of normal adduct 1, homoleptic tetraalkyl gallate 2 and heteroleptic gallate 3 . 


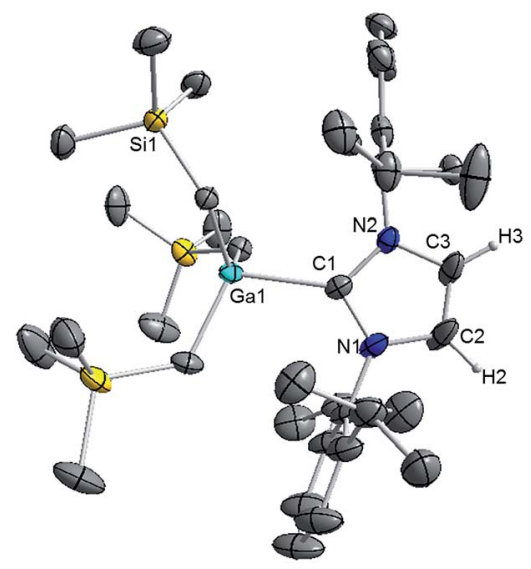

Fig. 1 Molecular structure of 1 with $50 \%$ probability displacement ellipsoids. All hydrogen atoms except those on the imidazole ring and minor disorder in one isopropyl group and one monosilyl group are omitted for clarity.

are connected by two bridging alkyl groups with the neutral NHC binding via its C2 (i.e. C1) position to lithium.

These findings show that under these reaction conditions the polar Li alkyl reagent preferentially co-complexes with $\mathrm{GaR}_{3}$, to yield $\left[\mathrm{LiGaR}_{4}\right]^{53}$ which is then trapped and stabilised by the neutral NHC ligand, instead of lithiating the carbene backbone. Clearly the $\mathrm{Ga}$ atom favours coordination of another $\mathrm{R}$ anion rather than a neutral IPr ligand. It should be noted that a similar reactivity has been reported for $\mathrm{IPr} \cdot \mathrm{Zn}^{t} \mathrm{Bu}_{2}$ with ${ }^{t} \mathrm{BuLi}$ in hexane, producing zincate complex $\left[\operatorname{IPr} \cdot \operatorname{LiZn}^{t} \mathrm{Bu}_{3}\right]^{54}$ Gallate 2 can also be prepared by reacting polymeric $\left[\left\{\mathrm{LiGaR}_{4}\right\}_{\infty}\right]$ with free IPr. Solution state studies of 2 were hindered by its poor solubility in arene solvents such as $\mathrm{C}_{6} \mathrm{D}_{6}$; whereas in coordinating THF, the adduct dissociates into free IPr and multi-THFsolvated $\mathrm{LiGaR}_{4}$, as evidenced by multinuclear NMR spectroscopy (see ESIt). Contrastingly, if the order of the monometallic reactants is reversed, by treating first IPr with LiR followed by the addition of gallium alkyl $\mathrm{GaR}_{3}$ in THF, heteroleptic $(\mathrm{THF})_{2} \mathrm{Li}$ $\left[: \mathrm{C}\left\{\left[\mathrm{N}\left(2,6-\mathrm{iPr}_{2} \mathrm{C}_{6} \mathrm{H}_{3}\right)\right]_{2} \mathrm{CHCGa}\left(\mathrm{CH}_{2} \mathrm{SiMe}_{3}\right)_{3}\right\}\right](3)$ was obtained in a $56 \%$ isolated yield. As shown in Scheme 1, 3 is the result of an

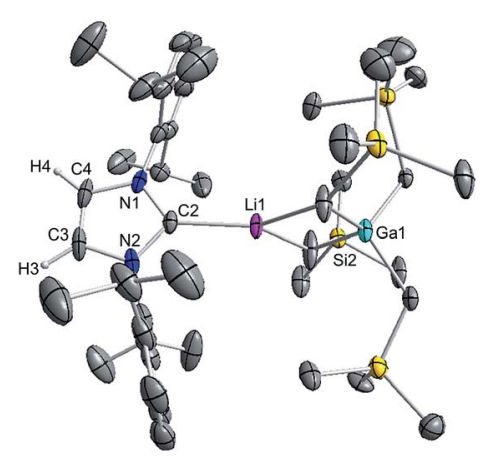

Fig. 2 Molecular structure of 2 with 30\% probability displacement ellipsoids. All $3 \mathrm{CH}_{2} \mathrm{SiMe}_{3}$ groups are disordered. Only one component of the disordered model is shown above. All hydrogen atoms except those on the imidazole ring have been omitted for clarity. indirect gallation process where IPr is first metallated at the C4 position by polar $\mathrm{LiR}^{36}$ which in turn undergoes transmetallation to the lower polarity $\mathrm{GaR}_{3}$. X-Ray crystallographic studies established the CIP structure of $\mathbf{3}$ where now the metals are connected by an anionic NHC which coordinates as an asymmetric bridge via its normal $\mathrm{C} 2$ position to $\mathrm{Li}$ and its abnormal C4 position to Ga (Fig. 3). The $\mathrm{C} 2-\mathrm{Li}$ (i.e. C1 in Fig. 3) distance is 2.093(5) $\AA$ which is similar to those reported for related complexes containing anionic NHC bridged in a similar $\mathrm{C} 2-\mathrm{Li} / \mathrm{C} 4-\mathrm{M}$ fashion to $\mathrm{Li} / \mathrm{Al}$ and $\mathrm{Li} / \mathrm{B}$ pairings. ${ }^{36,38}$

The Ga-C4 distance (i.e. C3 in Fig. 3) of 2.052(2) $\AA$ is close in value with $\mathrm{Ga}-\mathrm{C}_{\text {alkyl }}$ bonds (average $\left.2.028 \AA\right)^{55}$ and understandably it is significantly shorter (by $0.144 \AA$ ) to that found in the neutral $\mathrm{C} 2$ bound IPr adduct 1 . It is noteworthy, that unlike in $\mathbf{1}$ where a pyramidalization of Ga coordination sphere was evident (vide supra), in 3 gallium atom exhibits nearly ideal tetrahedral geometry with the average bond of $2.034 \AA$ and mean angle of $109.46^{\circ}$ (angles ranging from 105.68(9) ${ }^{\circ}$ to $\left.112.99(10)^{\circ}\right)$. This decrease in distortion around the metal centre can be attributed to the relief of the steric congestion of 3 when compared to 1 . From the NMR data in $d_{8}$-THF solutions (see ESI:), metallation of IPr was demonstrated by the large downfield chemical shift of the $\mathrm{C} 4$ resonance in the ${ }^{13} \mathrm{C}$ NMR spectrum (from $122.3 \mathrm{ppm}$ in free IPr to 155.1 in 3), as well as an informative singlet at $6.64 \mathrm{ppm}$ (integral $1 \mathrm{H}$ ) in the ${ }^{1} \mathrm{H}$ NMR spectrum of the imidazole $\mathrm{CH}$ (versus $7.19 \mathrm{ppm}$ in free IPr). In addition, a resonance in the ${ }^{13} \mathrm{C}$ NMR spectrum at $201.4 \mathrm{ppm}$ for carbenic $\mathrm{C} 2$ confirms the formation of a NHC complex. The loss of symmetry in the imidazole ring is evidenced in the ${ }^{1} \mathrm{H}$ and ${ }^{13} \mathrm{C}$ NMR spectra with the appearance of two distinct sets of Dipp signals.

\section{Abnormal NHC-Ga complexes via electrophilic interception}

Recent studies have shown that certain anionic NHC complexes, when treated with an electrophile can be transformed into neutral abnormal adducts. ${ }^{38,39}$ To explore this reactivity here, we treated 3 with a molar equivalent of MeOTf in toluene at $-78^{\circ} \mathrm{C}$. The reaction occurred with the formation of a white precipitate (presumably LiOTf) furnishing a neutral abnormal NHC-Ga complex $\left[\mathrm{CH}_{3} \mathrm{C}\left\{\left[\mathrm{N}\left(2,6-\mathrm{PPr}_{2} \mathrm{C}_{6} \mathrm{H}_{3}\right)\right]_{2} \mathrm{CHCGa}\left(\mathrm{CH}_{2} \mathrm{SiMe}_{3}\right)_{3}\right\}\right]$ (4) in a

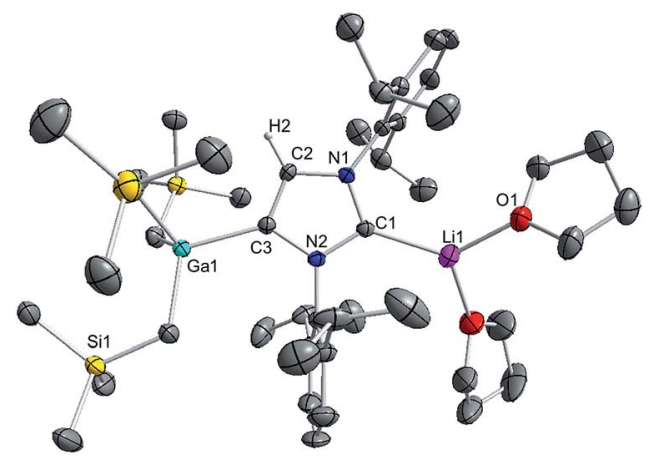

Fig. 3 Molecular structure of 3 with $50 \%$ probability displacement ellipsoids. All hydrogen atoms except $\mathrm{H} 2$ on the imidazole ring and minor disorder of THF ligand have been omitted for clarity. 
$68 \%$ yield (Scheme 2a). Complex 4 results from the selective $\mathrm{C} 2$ methylation of the anionic NHC leaving the $\mathrm{Ga}-\mathrm{C} 4$ bond intact (see ESI for experimental details and NMR spectroscopic characterisation).

The molecular structure of $\mathbf{4}$ was established by X-ray crystallographic studies (Fig. 4). The bond length of 1.538(6) A for ${ }^{\mathrm{C}} 2-\mathrm{C}_{\mathrm{Me}}$ (i.e. $\mathrm{C} 1-\mathrm{C} 4$ in Fig. 4) is consistent with a single bond, while the Ga-C4 (i.e. C3 in Fig. 4) bond length of 2.087(3) $\AA$ is only slightly elongated to that found in the anionic variant 3 (2.052(2) §), and significantly shorter than the Ga-C2 bond length in 1 (2.1960(16) ̊). Reflecting the formation of a neutral abnormal complex, the ${ }^{13} \mathrm{C}$ NMR spectrum of 4 shows a resonance at $161.2 \mathrm{ppm}$ for the $\mathrm{C} 4$ attached to $\mathrm{Ga}$ ( $v s .155 .1 \mathrm{ppm}$ in 3) whereas the methylated carbon (originally $\mathrm{C} 2$ carbenic position in 3) resonates significantly upfield at $145.0 \mathrm{ppm}$ in comparison with that observed for 3 (at $201.5 \mathrm{ppm}$ ).

The use of methanol as a quenching reagent resulted in clean conversion of 3 to the abnormal adduct $\operatorname{aIPr} \cdot \mathrm{GaR}_{3}$ (5) (Scheme $2 \mathrm{~b}$ ). Notably, $\mathbf{5}$ is also formed as a protonation product from the reaction of 3 with the imidazolium salt IMes $\cdot \mathrm{HCl}(1,3-$ bis-(2,4,6-trimethylphenyl)imidazolium chloride) (Scheme 2c). These findings not only demonstrate that the $\mathrm{C} 2$ position of 3 in the imidazole ring is its preferred basic site, but also the high strength of its $\mathrm{Ga}-\mathrm{C} 4$ bond as it is retained in 5. Furthermore in view of these results it appears that for the $\mathrm{GaR}_{3}$ fragment aIPr is a better ligand than the related normal IMes carbene (obtained from deprotonation of the imidazolium salt, Scheme 2c), as no ligand exchange occurs. Compound 5 was isolated as a crystalline solid in a $61 \%$ yield (see ESI + for experimental details and NMR spectroscopic studies) and its molecular structure was established by X-ray crystallography (Fig. 4).

The Ga-C4 bond length (i.e. Ga-C2 in Fig. 4) of 2.0759(16) in 5 is significantly shorter than the corresponding bond in the normal congener 1 (by $0.1201 \AA$ ), supporting previous studies which suggest that abnormal carbenes are stronger $\sigma$-donors, less sterically congested, and consequently are able to form stronger bonds with metal centres. In fact, as previously

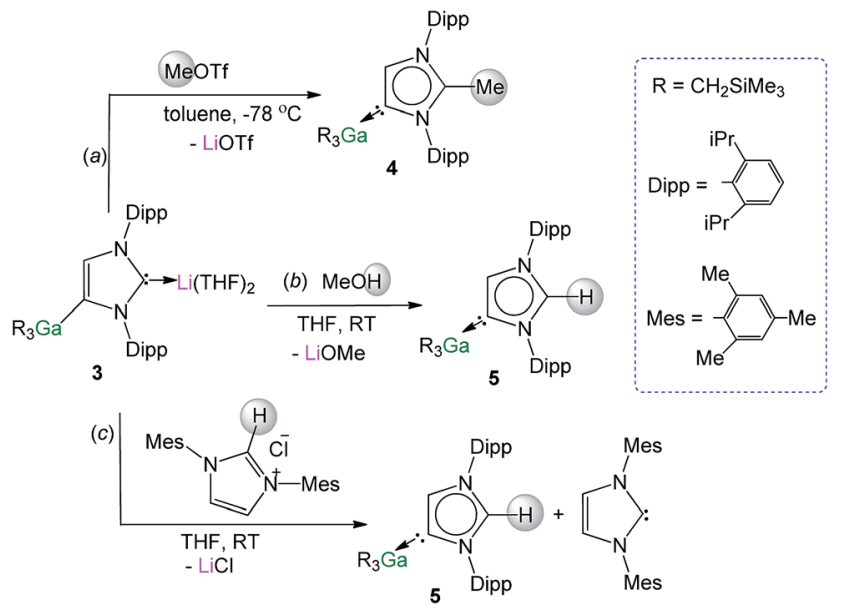

Scheme 2 Electrophilic interception of anionic NHC complex 3 with (a) MeOTf, (b) $\mathrm{MeOH}$ and (c) imidazolium salt IMes $\cdot \mathrm{HCl}$.

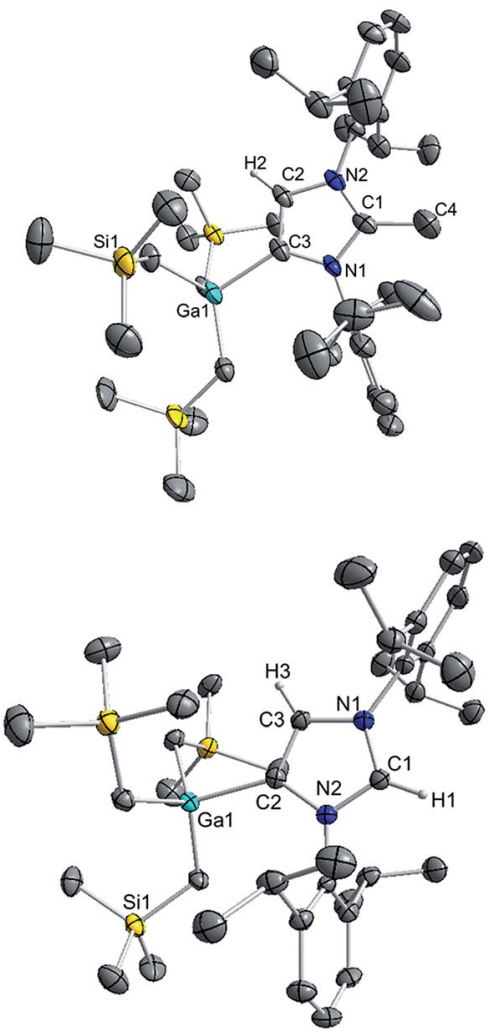

Fig. 4 Molecular structure of 4 (top) and 5 (bottom) with $50 \%$ probability displacement ellipsoids. Only one component of disordered groups is shown. All hydrogen atoms except those on the imidazole rings have been omitted for clarity.

discussed for 4, despite the neutral constitution of the aNHC ligand, the strength of this interaction is similar to that observed for the $\mathrm{Ga}-\mathrm{C}$ bond of the anionic carbene present in $\mathbf{3}$.

As mentioned above, the only example of an abnormal NHC Ga complex prior to this work has been reported by Robinson, ${ }^{39}$ where aIPr $\cdot \mathrm{GaCl}_{3}$ was formed while attempting the transmetallation of mixed lithium/boron anionic NHC complex with $\mathrm{GaCl}_{3}$. Interestingly the $\mathrm{Ga}-\mathrm{C}_{\text {carbene }}$ distance of this complex (1.978(3) A) differs only by $0.097 \AA$ to that found for 5 (2.0759(16) $\AA)$. This contrasts with the markedly different bond distances found when comparing the relevant normal isomer $\left(\mathrm{Ga}-\mathrm{C}_{\text {carbene }}\right.$ bond distance in IPr. $\mathrm{GaX}_{3}, 2.1960(16) \AA$ when $\mathrm{X}=\mathrm{R}$ (1); vs. 2.016(2) Å when $\mathrm{X}=\mathrm{Cl}$ ), hinting that in the abnormal systems, due to the increased steric space around the metal centre, the size of the anionic groups attached to Ga has a significantly smaller influence than in the normal adducts.

${ }^{1} \mathrm{H}$ NMR spectrum of 5 in $d_{8}$-THF showed a diagnostic singlet at 9.00 ppm belonging to the $\mathrm{H}$ attached to the $\mathrm{C} 2$ position of the carbene, whereas the remaining $\mathrm{H}$ in the imidazole ring resonates at $7.20 \mathrm{ppm}$. Similarly to that found in 4 , the ${ }^{13} \mathrm{C} N \mathrm{NM}$ spectrum shows two informative singlets at 162.8 and 139.1 ppm which can be assigned to $\mathrm{Ga}-\mathrm{C} 4$ and $\mathrm{NC}(\mathrm{H}) \mathrm{N}$ respectively.

Compound 5, along with 1 and $\mathbf{3}$ constitute a rare example of a series of normal, anionic and abnormal complexes incorporating the same metal-coligand partnership. ${ }^{56}$ Complementary DFT computational studies ${ }^{57}$ were undertaken on these three 
compounds employing the B3LYP method ${ }^{58}$ and the $6-311 \mathrm{G}^{* *}$ basis $\operatorname{set}^{59}$ (see ESI for details). Natural bond orbital (NBO) analysis $^{\mathbf{6 0}}$ of the optimised structures of $\mathrm{IPr} \cdot \mathrm{GaR}_{3}\left(\mathbf{I I I}_{\mathbf{I P r}}\right)$, aIPr. $\mathrm{GaR}_{3} \quad\left(\mathbf{I V}_{\mathbf{I P r}}\right) \quad$ and $\quad(\mathrm{THF})_{2} \mathrm{Li}\left[: \mathrm{C}\left\{\left[\mathrm{N}\left(2,6-\mathrm{iPr}_{2} \mathrm{C}_{6} \mathrm{H}_{3}\right)\right]_{2^{-}}\right.\right.$ $\left.\left.\mathrm{CHCGa}\left(\mathrm{CH}_{2} \mathrm{SiMe}_{3}\right)_{3}\right\}\right] \quad\left(\mathbf{V}_{\text {IPr }}\right)$ suggest considerable covalent character of the $\mathrm{Ga}-\mathrm{C}$ bonds. The Ga natural charges range from +1.43 to +1.35 and the Wiberg bond indices (WBIs) of the $\mathrm{Ga}-\mathrm{C}$ bonds range from 0.45-0.64. Contrastingly reflecting the more ionic nature of the $\mathrm{Li}-\mathrm{C}$ contact in $\mathbf{V}_{\mathbf{I P r}}$, the natural charge of $\mathrm{Li}$ is +0.88 and the WBI of the $\mathrm{Li}-\mathrm{C}$ is 0.08 . In agreement with our experimental findings the estimated Ga-C4 bonds in $\mathbf{I V}_{\mathbf{I P r}}$ and $\mathbf{V}_{\text {IPr }}$ are significantly shorter (2.146 and 2.054 $\AA$, WBIs $=0.56$ and 0.53 respectively) than the $\mathrm{Ga}-\mathrm{C} 2$ bond in the normal complex III IPr $_{\text {IPr }}(2.333 \AA$, WBI $=0.45)$, although it should be noted that for $\mathbf{I I I}_{\mathbf{I P r}}$ and $\mathbf{I V}_{\mathbf{I P r}}$, the strength of these $\mathrm{Ga}-\mathrm{C}$ bonds is somewhat underestimated (vide infra). A comparative natural charges analysis of these three models shows that while in bimetallic $\mathbf{V}_{\text {IPr }}$ the amount of electrons transfer to the $\mathrm{GaR}_{3}$ unit is 0.34 , in the case of adducts $\mathbf{I I I}_{\mathbf{I P r}}$ and $\mathbf{I V}_{\mathbf{I P r}}$ these values are 0.27 and 0.31 which is consistent with the neutral constitution of the NHC ligands.

Fig. 5 shows the highest occupied molecular orbitals (HOMOs) calculated for models $\mathbf{I I I}_{\text {IPr }}, \mathbf{I V}_{\text {IPr }}$ and $\mathbf{V}_{\text {IPr }}$ which in all cases correspond to the Ga-C bonding orbitals at the $\mathrm{CH}_{2}$ groups of the monosilyl ligands, involving also in the case of $\mathbf{V}_{\text {IPr }}$ the $\mathrm{C} 4$ of the anionic NHC. For this bimetallic system, these calculations contrast with those reported for the related anionic lithium dicarbene $\left[: \mathrm{C}\left\{\left[\mathrm{N}\left(2,6-\mathrm{iPr}_{2} \mathrm{C}_{6} \mathrm{H}_{3}\right)\right]_{2} \mathrm{CHCLi}(\mathrm{THF})\right\}\right]_{n}$ prepared by Robinson, ${ }^{36}$ whose HOMO and HOMO-2 correspond to the two strongly polarised $\mathrm{Li}-\mathrm{C}$ bonding orbitals at the $\mathrm{C} 2$ and $\mathrm{C} 4$ positions of the imidazole ring.

Interestingly, calculations on the regioisomeric structure of $\mathbf{V}_{\text {IPr }}$ with the positions of the $\left\{\mathrm{GaR}_{3}\right\}$ and $\left\{\mathrm{Li}(\mathrm{THF})_{2}\right\}^{+}$were reversed, giving rise to $\mathrm{Ga}-\mathrm{C} 2$ and $\mathrm{Li}-\mathrm{C} 4$ coordination modes (model $\mathbf{V I}_{\mathbf{I P r}}$ in ESI+) showed that this model is significantly less stable (by $11.7 \mathrm{kcal} \mathrm{mol}^{-1}$ ) which is consistent with the
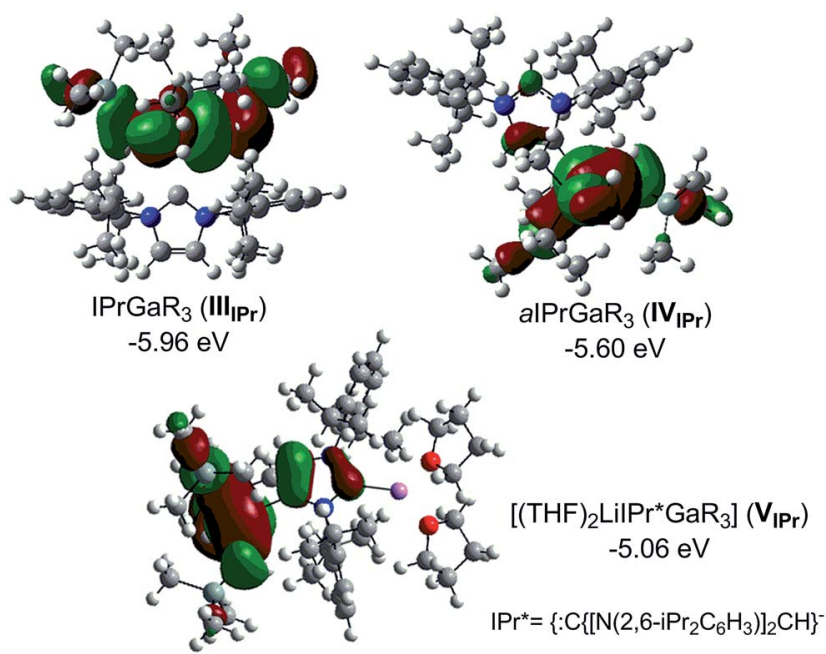

Fig. 5 Calculated molecular orbitals $\mathrm{HOMO}$ of models $\mathrm{III}_{\mathrm{IPr}}, \mathrm{IV}_{\mathrm{IPr}}$ and $\mathrm{V}_{\mathrm{IPr}}$. formation of a significantly weaker (longer) Ga-C bond (2.272 $\AA$ for $\mathbf{V I}_{\text {IPr }}$ vs. $2.054 \AA$ for $\mathbf{V}_{\text {IPr }}$, see ESIt for details).

\section{Normal to abnormal isomerization}

It is rare to find examples where both normal and abnormal isomers have been structurally characterised. ${ }^{61}$ Amongst them, intriguing studies from the groups of Layfield ${ }^{30}$ and Dagorne ${ }^{35}$ have shown that the systems IPr $\cdot \mathrm{Fe}(\mathrm{HMDS})_{2}$ and $\mathrm{I}^{t} \mathrm{Bu} \cdot \mathrm{AlMe}_{3}$ respectively, thermally isomerize to the relevant abnormal species although the possible reaction pathways for these transformations remain obscure. Similarly to our findings for complexes 1 and 5, in these $\mathrm{Fe}$ and $\mathrm{Al}$ examples, analysis of the metal-C distances have revealed that the abnormal NHCs bind more strongly to the metal centres than the isomeric normal carbenes. These studies also suggest the formation of the abnormal-NHC complex is thermodynamically controlled with steric factors strongly influencing isomerization processes. Since compound $\mathbf{5}$ was obtained using an indirect method (metallation/electrophilic interception), we pondered if such types of thermal rearrangement would also be in operation when 1 was heated in solution.

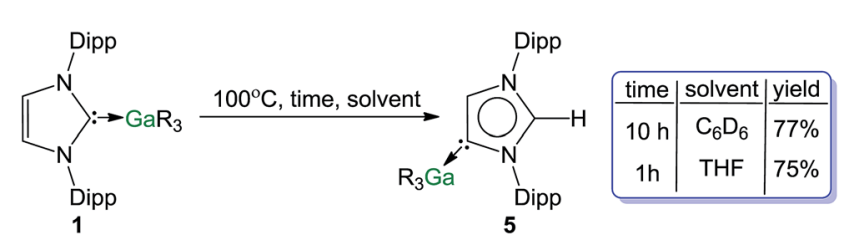

Scheme 3 Thermally-induced rearrangement of 1 into 5 .

Indeed, heating a $d_{6}$-benzene solution of $\mathbf{1}$ at $100{ }^{\circ} \mathrm{C}$ and monitoring progress by ${ }^{1} \mathrm{H}$ NMR, produced 5 in $77 \%$ yield after $10 \mathrm{~h}$. The reaction was greatly accelerated by using the more coordinating solvent $d_{8}$-THF (75\% conversion in only $1 \mathrm{~h}$ ) Scheme $3 .{ }^{62}$ Previous studies on ${ }^{t} \mathrm{Bu} \cdot \mathrm{AlMe}_{3}$ have shown that the isomerization is much faster using a Lewis donor solvent such as THF, hinting that dissociation (at least partially) of the carbene from the metal must play a significant role in the process. Interestingly no isomerization is observed for $\mathrm{IPr} \cdot \mathrm{GaCl}_{3}$ when using smaller and stronger Lewis acid $\mathrm{GaCl}_{3}$, as mentioned above, the carbene binds significantly more strongly to the Ga centre. Related to these findings, reflecting the relevance of the Lewis acidic character of the metal fragment, using the related alkyl compounds of other s-block metals such as $\mathrm{MgR}_{2} \cdot \operatorname{IPr}^{63}$ and $\mathrm{ZnR}_{2} \cdot \operatorname{IPr}^{64}(6)$ complexes $\left[\mathrm{R}=\mathrm{CH}_{2} \mathrm{SiMe}_{3}\right]$ no rearrangements were observed after $72 \mathrm{~h}$ at $100{ }^{\circ} \mathrm{C}$.

The effect of the steric bulk of the substituents on the NHC ligand was assessed. IMes $\cdot \mathrm{GaR}_{3}$ (7), containing the less bulky 1,3-bis-(2,4,6-trimethylphenyl)imidazol-2-ylidene (IMes) ${ }^{65}$ carbene, rearranges at a significantly slower rate than $\mathbf{1}$, showing after 30 hours at $100{ }^{\circ} \mathrm{C}$ in $d_{8}$-THF a modest $8 \%$ conversion to its abnormal isomer. Contrastingly, 1,3-bis(tert-butyl)imidazol-2ylidene $\left(I^{t} \mathrm{Bu}\right)$ failed to form a normal adduct with $\mathrm{GaR}_{3}$, furnishing instead only the abnormal isomer $\mathrm{aI}^{t} \mathrm{Bu} \cdot \mathrm{GaR}_{3}(8)$ at 
room temperature within one hour. This reactivity contrasts with that reported for the iron complex I ${ }^{t} \mathrm{Bu} \cdot \mathrm{Fe}(\mathrm{HMDS})_{2}$ which undergoes thermal decomposition, ${ }^{30}$ and appears to be more in line with that reported by Tamm for the $\mathrm{B}\left(\mathrm{C}_{6} \mathrm{~F}_{5}\right)_{3} / \mathrm{I}^{t} \mathrm{Bu}$ frustrated Lewis pair system (FLP) which has been used for the activation of small molecules such as $\mathrm{H}_{2}$ or alkynes. Although the two components fail to give an isolable normal complex, in the absence of other reactive substrates, the irreversible formation of the relevant abnormal carbene-borane adduct is observed..$^{32-34}$

\section{DFT calculations ${ }^{57}$}

Encouraged by the formation of several aNHC-Ga complexes using this approach, we performed theoretical calculations at the DFT level employing the B3LYP method ${ }^{58}$ and the $6-311 \mathrm{G}^{* *}$ basis $\operatorname{set}^{59}$ to optimize structures and to gain new insights into the thermodynamics involved in these processes. A comparison of geometrical parameters of optimised structures $\mathrm{IPr} \cdot \mathrm{GaR}_{3}$ $\left(\right.$ III $\left._{\text {IPr }}\right)$ and $\operatorname{aIPr} \cdot \mathrm{GaR}_{3}\left(\mathbf{I V}_{\text {IPr }}\right)$ shows in general good agreement with those found experimentally from the X-ray determinations of 1 and 5 respectively (see Fig. 6 and Table S3 ) although for both models there is a slight underestimation of the strength of the $\mathrm{Ga}-\mathrm{C}_{\text {carbene }}$ interaction $\left(\Delta\left[d(\mathrm{Ga}-\mathrm{C})_{\text {calc }}-d(\mathrm{Ga}-\mathrm{C})_{\exp }\right)=0.137\right.$ and $0.070 \AA$ for $\mathbf{1}$ and 5 respectively). Interestingly model $\mathbf{I V}_{\mathbf{I P r}}$ was computed to be more stable than III $_{\text {IPr }}$ by just $1.5 \mathrm{kcal} \mathrm{mol}^{-1}$. Using the same level of theory we also found that free aIPr is $16.2 \mathrm{kcal} \mathrm{mol}^{-1}$ less stable than its normal isomer IPr. ${ }^{66}$ Notably, the dissociation energy of $\mathbf{I V}_{\mathbf{I P r}}$ was found to be $17.7 \mathrm{kcal} \mathrm{mol}^{-1}$ higher than for III IPr $_{\text {(Fig. 7), in agreement with our experimental }}$ findings which suggest a greater donor ability of the abnormal $\mathrm{NHC}$ ligand for the $\mathrm{GaR}_{3}$ fragment when compared to its normal isomer.

These studies were extended to the related carbenes IMes and $I^{t} \mathrm{Bu}$. For IMes, which is less sterically demanding than IPr, the order of stability of $\mathbf{I I I}_{\mathbf{I M e s}}$ and $\mathbf{I V}_{\mathbf{I M e s}}$ is reversed, with the normal isomer $\mathbf{I I I}_{\text {IMes }}$ being $2.1 \mathrm{kcal} \mathrm{mol}^{-1}$ more stable (see ESI $\$)$. Interestingly, the energy difference between free aIMes

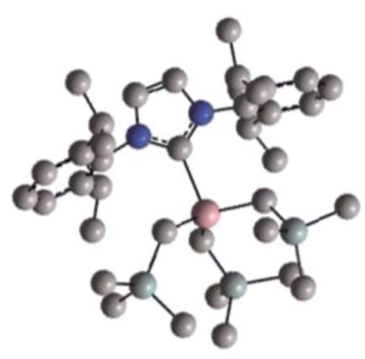

$\mathrm{IPr} . \mathrm{GaR}_{3}\left(\mathrm{III}_{\mathrm{IPr}}\right)$ $\left(+1.5 \mathrm{kcal} \mathrm{mol}^{-1}\right)$

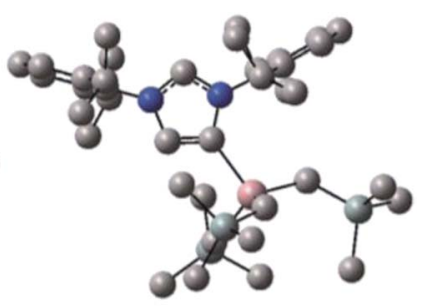

$\operatorname{alPr} \mathrm{GaR}_{3}\left(\mathrm{IV}_{\mathrm{IPr}}\right)$ $\left(0.0 \mathrm{kcal} \mathrm{mol}^{-1}\right)$

\begin{tabular}{|c|c|c|}
\hline Model & $\mathrm{IIII}_{\mathrm{IPr}}$ & $\mathrm{IV}_{\mathrm{IPr}}$ \\
\hline $\mathrm{Ga}-\mathrm{C}_{\mathrm{NHC}}(\AA)$ (calc) & 2.333 & 2.146 \\
\hline $\mathrm{Ga}-\mathrm{C}_{\mathrm{NHC}}(\AA)(\exp )$ & $2.1960(16)$ & $2.0759(16)$ \\
\hline
\end{tabular}

Fig. 6 Modelled structures and relative energies of $\mathrm{NHC}$-adducts $\mathrm{III}_{\operatorname{IPr}}$ and $\mathrm{IV} \mathrm{IPr}$. and IMes of $+16.8 \mathrm{kcal} \mathrm{mol}^{-1}$ is almost identical to that for free aIPr and IPr. As shown in Fig. 7, the calculated values for the dissociation energies of $\mathbf{I I I}_{\mathbf{I M e s}}$ and $\mathbf{I V}_{\mathbf{I M e s}}$ follow the same trend as described for the IPr complexes, although now the dissociation of IMes $\cdot \mathrm{GaR}_{3}$ ( III IMes $_{\mathbf{I M}}$ ) is noticeably more endothermic (by $6.1 \mathrm{kcal} \mathrm{mol}{ }^{-1}$ ) than in $\mathrm{IPr} \cdot \mathrm{GaR}_{3}\left(\mathbf{I I I}_{\mathbf{I P r}}\right)$. These subtle but significant changes in the energy values could explain the lower conversions observed experimentally when IMes $\cdot \mathrm{GaR}_{3}(7)$ is heated in $d_{8}$-THF (max yield $8 \%$ for aIMes $\cdot \mathrm{GaR}_{3}$, vide supra), as the dissociation of IMes $\cdot \mathrm{GaR}_{3}$ is more thermodynamically challenging.

A more dramatic effect is observed for $\mathrm{I}^{t} \mathrm{Bu}$, which containing aliphatic ${ }^{t} \mathrm{Bu}$ substituents, is significantly bulkier than IPr, more basic and therefore a better donor, from an electronic perspective. ${ }^{67}$ Notably attempts to optimize the structure of $\mathrm{I}^{t} \mathrm{Bu} \cdot \mathrm{GaR}_{3}$ were unsuccessful, as all the obtained models showed no stabilisation compared to the separate constituents $\mathrm{I}^{t} \mathrm{Bu}$ and $\mathrm{GaR}_{3}$. This lack of coordination between $\mathrm{I}^{t} \mathrm{Bu}$ and $\mathrm{GaR}_{3}$ can best be explained by steric incompatibility of ${ }^{t} \mathrm{Bu}$ and monosilyl groups and supports our experimental findings that when $\mathrm{I}^{t} \mathrm{Bu}$ and $\mathrm{GaR}_{3}$ are mixed at $\mathrm{RT} \mathrm{II}^{t} \mathrm{Bu} \cdot \mathrm{GaR}_{3}(\mathbf{8})$ is formed. The dissociation energy for this abnormal complex was found to be $+23.9 \mathrm{kcal} \mathrm{mol}^{-1}$ (Fig. 7)

Collectively these computational results not only offer further support for the greater donor ability of abnormal NHC ligands compared to their normal isomers but also highlight the crucial role that the steric profile plays in these isomerization processes. Thus, in the case of IMes, the less bulky of the
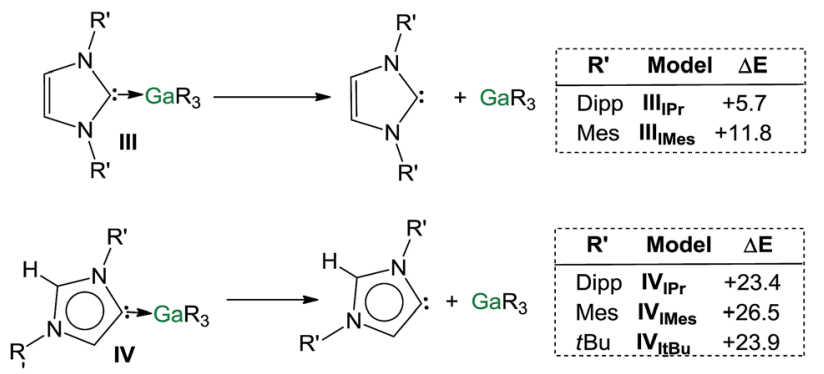

Fig. 7 Estimated dissociation energies ( $\mathrm{kcal} \mathrm{mol}^{-1}$ ) of complexes III and IV ( $R=$ Dipp, Mes, $\left.{ }^{t} \mathrm{Bu}\right)$.

Table 1 Initial rates for isomerization of 1 into 5 in $d_{8}$-THF at $323 \mathrm{~K}$ and at given initial concentrations of $1, \operatorname{IPr}$ and $\mathrm{GaR}_{3}$

\begin{tabular}{lllll}
\hline Entry & {$[1] \mathrm{M}$} & {$[\mathrm{IPr}](\mathrm{M})$} & {$\left[\mathrm{GaR}_{3}\right](\mathrm{M})$} & $r_{\mathrm{o}} \times 10^{6}\left(\mathrm{Ms}^{-1}\right)$ \\
\hline 1 & 0.17 & & & $1.95 \pm 0.08$ \\
2 & 0.29 & & & $3.26 \pm 0.08$ \\
3 & 0.37 & & & $4.81 \pm 0.03$ \\
4 & 0.42 & & & $5.04 \pm 0.04$ \\
5 & 0.29 & 0.07 & & $4.67 \pm 0.05$ \\
6 & 0.29 & 0.15 & & $5.71 \pm 0.15$ \\
7 & 0.29 & 0.26 & & $6.40 \pm 0.09$ \\
8 & 0.32 & & 0.16 & $3.39 \pm 0.04$ \\
9 & 0.32 & & 0.31 & $2.30 \pm 0.02$ \\
10 & 0.32 & & 0.42 & $2.06 \pm 0.04$
\end{tabular}



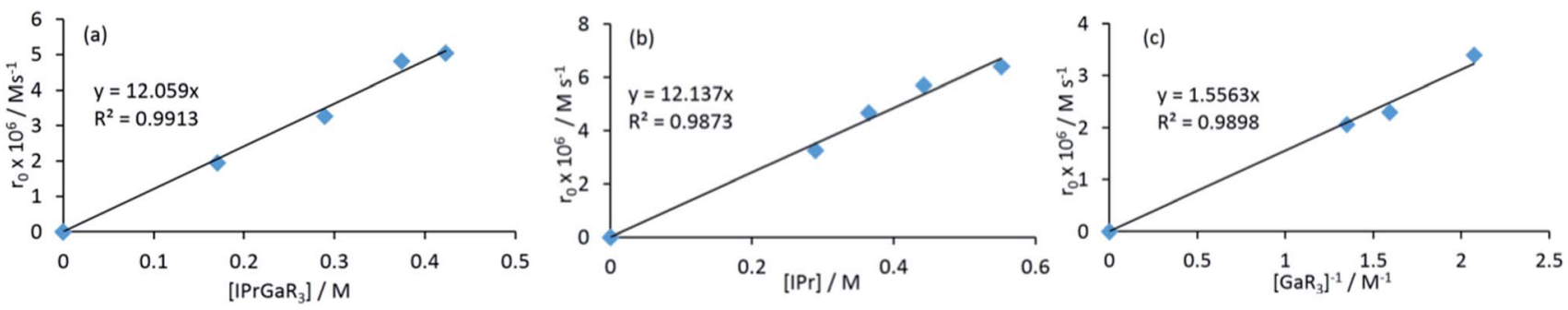

Fig. 8 Initial rates versus concentration of (a) [1], (b) [IPr] and (c) $\left[\mathrm{GaR}_{3}\right]^{-1}$.

carbenes investigated, it becomes slightly endothermic, whereas for $\mathrm{IPr}$ and $\mathrm{I}^{t} \mathrm{Bu}$, the formation of the abnormal complexes is thermodynamically favoured by -1.5 and $-6.6 \mathrm{kcal} \mathrm{mol}^{-1}$ respectively, in accord with their sizes.

\section{Mechanistic implications}

Both computational and spectroscopic studies suggest that the isomerization of $\mathbf{1}$ into $\mathbf{5}$ may involve a dissociative step. Supporting this assumption, when a mixture of $\mathbf{1}$ and $\mathrm{GaR}_{3}$ (two equivalents) was heated at $100{ }^{\circ} \mathrm{C}$ in $d_{8}$-THF, the formation of 5 becomes significantly slower $(46 \%$ conversion observed after $1 \mathrm{~h}$ ), which can be rationalised in terms of the effect that the excess of this reagent will have in the equilibrium depicted in eqn (1), namely shift it towards the left.

$$
\mathrm{GaR}_{3}-\mathrm{IPr} \rightleftharpoons \mathrm{GaR}_{3}+\mathrm{IPr}
$$

Contrastingly, when the reaction is carried out using an excess of IPr ( 2 equivalents), the isomerization process occurs significantly faster (90\% after 30 minutes). In order to shed some light on the mechanism involved in this isomerization process, kinetic analysis of a NMR scale reaction $([1]=0.22 \mathrm{M})$ performed at $100{ }^{\circ} \mathrm{C}$ in $d_{8}$-THF revealed a pseudo-zeroth-order kinetics over a period of two-half lives (64\% conversion). ${ }^{62}$ An identical experiment using $\operatorname{IPr}^{\mathrm{D}} \cdot \mathrm{GaR}_{3}\left(\mathbf{1}^{\mathrm{D}}\right)\left(\operatorname{IPr}^{\mathrm{D}}=1,3\right.$-bis(2,6-diisopropylphenyl)-4,5-dideutero-imidazolin-2-ylidene) ${ }^{68}$ allowed the comparison of the subsequent zero order rate constants,

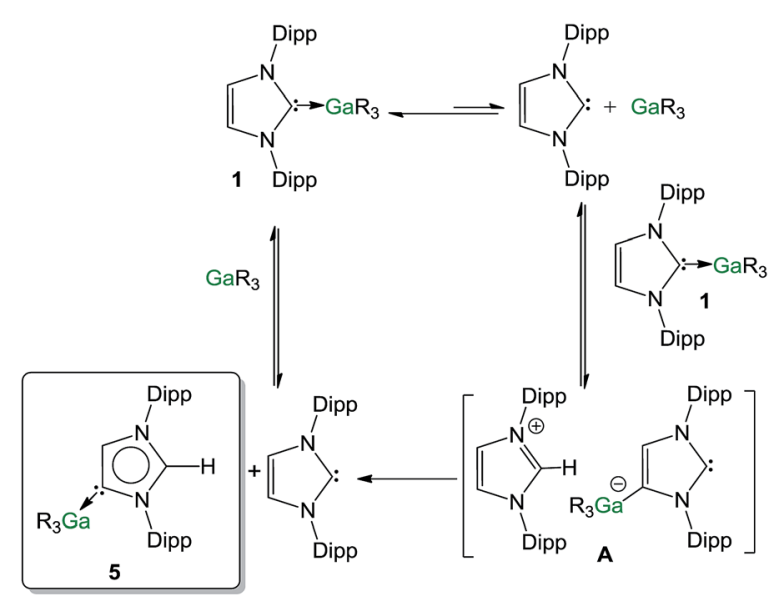

Fig. 9 Proposed mechanism for the isomerisation of 1 into 5. revealing no observable KIE (see ESI† for details). These findings suggest that the bond cleavage of the $\mathrm{C}_{4}-\mathrm{H}$ in the IPr ligand is unlikely to be rate-determining in the isomerization process. Encouraged by these findings we chose to study further the mechanism of this process using the method of initial rates. ${ }^{69,70}$ Table 1 shows the results of the initial rate experiments conducted in a sealed NMR tube $\left(50{ }^{\circ} \mathrm{C}, 1, d_{8}\right.$-THF $)$. These data were fitted to the approximately linear region of the formation of $\mathbf{5}$ restricted to conversions of $5-7 \%$ in order to calculate the initial rate, $r_{\mathrm{o}}$ of the reaction. Entries 1-4 demonstrate a first-order dependence on the concentration of complex 1 while Fig. 8a shows this correlation graphically. A first-order dependence is also observed for the concentration of IPr as shown in entries 2, 5-7 and Fig. 8b, which is consistent with the dissociative step previously discussed and the involvement of free IPr in the isomerization process. By contrast to IPr, a negative order of -1 is observed for the concentration of $\mathrm{GaR}_{3}$ (entries 8-10, Fig. 8c). A plausible interpretation of these results is that the isomerization process takes place by the partial dissociation of $\mathbf{1}$ (which appears to be the rate-determining step of the reaction $)^{71}$ to form free IPr that in turn can activate the $\mathrm{H}$ atom from the backbone of the NHC ligand coordinated to Ga in complex $1 .^{72}$ This proposed modus operandi is similar to that described for the activation of small molecules such as acetylenes, $\mathrm{H}_{2}$ or amines using NHC/borane FLP systems, that, as mentioned before, in the absence of another substrate form the relevant abnormal aNHC-BAr ${ }_{3}$ adducts $\left(\mathrm{Ar}=\mathrm{C}_{6} \mathrm{~F}_{5}\right.$ or $\left.\mathrm{XyF}_{6}\right) .^{32-34,73}$ This could lead to the formation of the transient ion-pair species $[\mathrm{IPr}-\mathrm{H}]^{+}\left[\mathrm{IPr}^{*} \mathrm{GaR}_{3}\right]^{-}$(A) (IPr* = :C $\left\{\left[\mathrm{N}\left(2,6-\mathrm{iPr}_{2} \mathrm{C}_{6} \mathrm{H}_{3}\right)\right]_{2} \mathrm{CHC}\right)$, comprising an imidazolium cation and an NHC-gallate containing an anionic NHC (which on the basis of the constitution of lithium gallate 3 , it could be expected to have its Ga center coordinated to the $\mathrm{C} 4$ position) (Fig. 9). This reactivity can be interpreted in terms of "thermally induced frustration," a concept recently introduced by Pápai which refers to the thermal activation of strained dative bonds of bulky Lewis donor-acceptor pairs. ${ }^{74}$ Intermediate A will evolve fast with the irreversible formation of abnormal complex $\mathbf{5}$ and the regeneration of free IPr. This proposed behaviour mirrors that described in Scheme 2c, for the protonation of the anionic carbene present in 3 by the imidazolium salt IMes $\cdot \mathrm{HCl}$, which occurs at the $\mathrm{C} 2$ position, forming neutral $\mathrm{aIPr} \cdot \mathrm{GaR}_{3}$ and free IMes. Since IPr is regenerated at the end of the process, it can then be envisaged that under the conditions studied it acts as a catalyst in the isomerization process. 


\section{Conclusions}

Progressing main-group NHC chemistry, this systematic study of the synthesis and stability of abnormal NHC-gallium complexes has demonstrated two alternative and efficient methodologies to access $\operatorname{aIPr} \cdot \mathrm{GaR}_{3}(5)$. Studies investigating the synthesis of anionic NHC complexes have shown that the functionalization of the imidazole backbone can be achieved by sequentially treating IPr with the polar organometallic reagent LiR followed by $\mathrm{GaR}_{3}$ addition (indirect stepwise gallation), to afford heteroleptic gallate $(\mathrm{THF})_{2} \mathrm{Li}\left[\mathrm{C}\left\{\left[\mathrm{N}\left(2,6-\mathrm{iPr}_{2} \mathrm{C}_{6} \mathrm{H}_{3}\right)\right]_{2}{ }^{-}\right.\right.$ $\left.\left.\mathrm{CHCGa}\left(\mathrm{CH}_{2} \mathrm{SiMe}_{3}\right)_{3}\right\}\right]$ (3). Electrophilic interception of 3 with MeOTf or the imidazolium salt IMes $\cdot \mathrm{HCl}$ led to the isolation of neutral abnormal NHC (aNHC) complexes $\left[\mathrm{CH}_{3} \mathrm{C}\left\{\left[\mathrm{N}\left(2,6-\mathrm{iPr}_{2}-\right.\right.\right.\right.$ $\left.\left.\left.\left.\mathrm{C}_{6} \mathrm{H}_{3}\right)\right]_{2} \mathrm{CHCGa}\left(\mathrm{CH}_{2} \mathrm{SiMe}_{3}\right)_{3}\right\}\right]$ (4) and aIPr. $\mathrm{GaR}_{3}$ (5). These studies disclose the preference of the anionic IPr ligand present in 3 to react with these electrophiles via its $\mathrm{C} 2$ position, leaving its $\mathrm{Ga}-\mathrm{C} 4$ interaction intact. Compound 5 can also be accessed by a thermally induced rearrangement of its normal isomer 1 . NMR spectroscopic studies coupled with theoretical calculations have revealed the importance of the donor ability of the solvent used in these thermal isomerization processes as well as the steric bulk of the substituents on the $\mathrm{N}$ atoms of the NHC ligands and the $\mathrm{Ga}$ reagent, suggesting that the relief of the steric hindrance by forming an abnormal complex is one of the main driving forces behind these rearrangements and hinting at the potential FLP reactivity that these systems may exhibit. Mechanistic studies intimate that these processes occur via a rate-determining dissociative step, supporting the formation of free NHC, which in turn can catalyse the isomerization process.

\section{Acknowledgements}

We thank the Royal Society, and the European Research Council (ERC) for their generous sponsorship of this research. We also thank Professor Robert E. Mulvey, Dr David. J. Nelson (University of Strathclyde) and Professor Michael S. Hill (University of Bath) for their insightful comments, Mr Alexander Clunie for his assistance in the CHN analysis of highly air and moisture sensitive compounds and Mr Craig Irving for his help with NMR spectroscopy experiments.

\section{Notes and references}

1 (a) D. Bourissou, O. Guerret, F. P. Gabbaï and G. Bertrand, Chem. Rev., 2010, 100, 39; (b) M. Albrecht, Science, 2009, 326, 532; (c) W. Kirmse, Angew. Chem., Int. Ed., 2010, 49, 8798; (d) N-Heterocyclic Carbenes in Transition Metal Catalysis and Organocatalysis, ed. C. S. J. Cazin, Springer, Netherlands, 2010; (e) N-Heterocyclic Carbenes: Effective Tools for Organometallics Synthesis, ed. S. P. Nolan, Wiley VCH, 2014.

2 A. J. Arduengo III, R. L. Harlow and M. Kline, J. Am. Chem. Soc., 1991, 113, 361.

3 A. J. Arduengo III, H. V. Rasika Dias, D. A. Dixon, R. L. Harlow, W. T. Klooster and T. F. Koetzle, J. Am. Chem. Soc., 1994, 116, 6812.
4 M. Scholl, S. Ding, C. W. Lee and R. H. Grubbs, Org. Lett., 1999, 1, 953.

5 W. Hermann, Angew. Chem., Int. Ed., 2002, 41, 1290.

6 C. Valente, S. Calimsiz, K. H. Hoi, D. Mallik, M. Sayah and M. G. Organ, Angew. Chem., Int. Ed., 2012, 51, 3314.

7 S. P. Nolan, N-Heterocyclic Carbenes in Synthesis, Wiley-VCH, Weinheim, 2006.

8 S. Diez-Gonzalez, N. Marion and S. P. Nolan, Chem. Rev., 2009, 109, 3612.

9 D. Enders, O. Niemeier and A. Henseler, Chem. Rev., 2007, 107, 5606.

10 Y. Wang, Y. Xie, P. Wei, R. B. King, H. F. Schaefer III, P. v. R. Schleyer and G. H. Robinson, Science, 2008, 321, 1069.

11 A. Sidiropoulos, C. Jones, A. Stasch, S. Klein and G. Frenking, Angew. Chem., Int. Ed., 2009, 48, 9701.

12 R. S. Ghadwal, H. W. Roesky, S. Merkel, J. Henn and D. Stalke, Angew. Chem., Int. Ed., 2009, 48, 5683.

13 C. Jones, A. Sidiropoulos, N. Holzmann, G. Frenking and A. Stasch, Chem. Commun., 2012, 48, 9855.

14 Y. Wang, Y. Xie, P. Wei, R. B. King, H. F. Schafer III, P. v. R. Schleyer and G. H. Robinson, J. Am. Chem. Soc., 2008, 130, 14970.

15 M. Y. Abraham, Y. Wang, Y. Xie, P. Wei, H. F. Schaefer III, P. v. R. Schleyer and G. H. Robinson, Chem.-Eur. J., 2010, 16, 432 .

16 H. Braunschweig, R. D. Dewhurst, K. Hammond, J. Mies, K. Radacki and A. Vargas, Science, 2012, 336, 1420.

17 For excellent overviews of the area see: (a) R. S. Ghadwal, R. Azhakar and H. W. Roesky, Acc. Chem. Res., 2013, 46, 444; (b) Y. Wang and G. H. Robinson, Inorg. Chem., 2011, 50, 12326.

18 L. J. Hounjet and D. W. Stephan, Org. Process Res. Dev., 2014, 18, 385 .

19 D. W. Stephan, Acc. Chem. Res., 2015, 48, 306.

20 R. Tonner, G. Heydenrych and G. Frenking, Chem.-Asian J., 2007, 2, 1555.

21 S. Gründemann, A. Kovacevic, M. Albrecht, J. W. Faller and R. H. Crabtree, Chem. Commun., 2001, 2274.

22 S. Gründemann, A. Kovacevic, M. Albrecht, J. W. Faller and R. H. Crabtree, J. Am. Chem. Soc., 2002, 124, 10473.

23 L. N. Appelhans, D. Zuccaccia, A. Kovacevic, A. R. Chianese, J. R. Miecznikowski, A. Macchioni, E. Clot, O. Eisenstein and R. H. Crabtree, J. Am. Chem. Soc., 2005, 127, 16299.

24 (a) P. L. Arnold and S. Pearson, Coord. Chem. Rev., 2007, 251, 596; (b) O. Schuster, L. Yang, H. G. Raubenheimer and M. Albrecht, Chem. Rev., 2009, 109, 3445; (c) R. H. Crabtree, Coord. Chem. Rev., 2013, 257, 755.

25 E. Aldeco-Perez, A. J. Rosenthal, B. Donnadieu, P. Parameswaran, G. Frenking and G. Bertrand, Science, 2009, 326, 556.

26 Related to these findings, Ghadwal et al. have recently reported a Pd catalysed methodology to access C2-arylated imidazolium salts which can be used as precursors to prepare abnormal-NHC Cu complexes, see: R. S. Ghadwal, S. O. Reichmann and R. Herbst-Irmer, Chem.-Eur. J., 2015, 21, 4247. 
27 J. B. Waters and J. M. Goicoechea, Coord. Chem. Rev., 2015, 293-294, 80.

28 M. Heckenroth, E. Kluser, A. Neels and M. Albrecht, Angew. Chem., Int. Ed., 2007, 46, 6293.

29 The Tolman Electronic Parameter (TEP) has been employed in transition metal complexes to evaluate the electron donating abilities of normal and abnormal NHC's, see for example: R. A. Kely III, H. Clavier, S. Giudice, N. M. Scott, E. D. Stevens, J. Bordner, I. Samardjiev, C. D. Hoff, L. Cavallo and S. P. Nolan, Organometallics, 2008, 27, 202.

30 B. M. Day, T. Pugh, D. Hendriks, C. F. Guerra, D. J. Evans, F. M. Bickelhaupt and R. A. Layfield, J. Am. Chem. Soc., 2013, 135, 13338.

31 T. W. Graham, K. A. Udachin and A. J. Carty, Chem. Commun., 2006, 2699.

32 D. Holschummacher, T. Bannenberg, C. G. Hrib, P. G. Jones and M. Tamm, Angew. Chem., Int. Ed., 2008, 47, 7428.

33 D. Holschummacher, T. Bannenberg, K. Ibrom, C. G. Daniliuc, P. G. Jones and M. Tamm, Dalton Trans., 2010, 39, 10590.

34 E. L. Kolychev, T. Bannenberg, M. Freytag, C. G. Daniliuc, P. G. Jones and M. Tamm, Chem.-Eur. J., 2012, 18, 16938.

35 A. L. Schmitt, G. Schnee, R. Welter and S. Dagorne, Chem. Commun., 2010, 46, 2480.

36 Y. Wang, Y. Xie, M. Y. Abraham, P. Wei, H. F. Schaefer III, P. v. R. Schleyer and G. H. Robinson, J. Am. Chem. Soc., 2010, 132, 14370.

37 Y. Wang, Y. Xie, M. Y. Abraham, R. G. Gilliard Jr, P. Wei, C. F. Campana, H. F. Schaefer III, P. v. R. Schleyer and G. H. Robinson, Angew. Chem., Int. Ed., 2012, 51, 10173.

38 Y. Wang, M. Y. Abraham, R. G. Gilliard Jr, P. Wei, J. C. Smith and G. H. Robinson, Organometallics, 2012, 31, 791.

39 M. Chen, Y. Wang, R. J. Gilliard Jr, P. Wei, N. A. Schwartz and G. H. Robinson, Dalton Trans., 2014, 43, 14211.

40 (a) A. El-Hellani and V. Lavallo, Angew. Chem., Int. Ed., 2014, 53, 4489; (b) M. J. Asay, S. P. Fisher, S. E. Lee, D. Borchardt, F. S. Tham and V. Lavallo, Chem. Commun., 2015, 51, 5359.

41 X.-W. Li, J. Su and G. H. Robinson, Chem. Commun., 1996, 2683.

42 N. Marion, E. C. Escudero-Adan, J. Benet-Buchholz, E. D. Stevens, L. Fensterbank, M. Malacria and S. P. Nolan, Organometallics, 2007, 26, 3256.

43 C. Fliedel, G. Schnee, T. Aviles and S. Dagorne, Coord. Chem. Rev., 2014, 275, 63.

44 A. Higelin, S. Keller, C. Göhringer, C. Jones and I. Krossing, Angew. Chem., Int. Ed., 2013, 52, 4941.

45 M. L. Cole, S. K. Furfari and M. Kloth, J. Organomet. Chem., 2009, 694, 2934.

46 S. Tang, J. Monot, A. El-Hallani, B. Michelet, R. Guillot, C. Bour and V. Gandon, Chem.-Eur. J., 2012, 18, 10239.

47 C. Bour, J. Monot, S. Tang, R. Guillot, J. Farjon and V. Gandon, Organometallics, 2014, 33, 594.

48 O. T. Beachley Jr and R. G. Simmons, Inorg. Chem., 1980, 19, 1021.

49 M. U. Kramer, D. Robert, Y. Nakajima, U. Englert, T. P. Spaniol and J. Okuda, Eur. J. Inorg. Chem., 2007, 5, 665.
50 Two singlets are observed for the monosilyl group at -0.95 $\left(\mathrm{Ga}-\mathrm{CH}_{2}\right)$ and $0.18 \mathrm{ppm}\left(\mathrm{Si}\left(\mathrm{CH}_{3}\right)_{3}\right)$ contrasting with the ${ }^{1} \mathrm{H}$ NMR spectrum of $\mathrm{GaR}_{3}$ in the same solvent where both signals accidentally overlap displaying a singlet at $0.13 \mathrm{ppm}$.

51 A. Jana, R. Azhakar, G. Tavčar, H. W. Roesky, I. Objartel and D. Stalke, Eur. J. Inorg. Chem., 2011, 25, 3686.

52 For both 2 and 4 the three monosilyl groups were found to be disordered. This obviously compromises any discussion of structural details such as bond distances, however in each case the crystallographic analysis does establish the gross composition and conformation of the molecular complexes.

53 The structure of this unsolvated lithium gallate has been reported recently, see: D. R. Armstrong, E. Brammer, T. Cadenbach, E. Hevia and A. R. Kennedy, Organometallics, 2013, 32, 480.

54 D. R. Armstrong, S. E. Baillie, V. L. Blair, N. G. Chabloz, J. Diez, J. Garcia-Alvarez, A. R. Kennedy, S. D. Robertson and E. Hevia, Chem. Sci., 2013, 4, 4259.

55 All four $\mathrm{Ga}-\mathrm{C}$ bonds are similar in length ranging from 2.013(2) $\AA$ to 2.052(2) $\AA$ (average 2.034 $\AA$ ) which is in good agreement with other tetra-coordinated gallate species such as $\left\{[\text { K-dibenzo-18-c-6 }]^{+}\left[\mathrm{Ga}\left(\eta^{1}-\mathrm{C}_{3} \mathrm{H}_{5}\right)_{4}\right]^{-}\right\} \quad(2.029 \quad \AA$ mean) see ref: C. Lichtenberg, T. P. Spaniol and J. Okuda, Inorg. Chem., 2012, 51, 2254.

56 As far as we can ascertain there are only two other examples containing the $\mathrm{BEt}_{3}$ (see ref. 38 and 39 and Y. Yamaguchi, T. Kashiwabara, K. Ogata, Y. Miura, Y. Nakamura, K. Kobayashi and T. Ito, Chem. Commun., 2004, 2160) and $\mathrm{B}\left(\mathrm{C}_{6} \mathrm{~F}_{5}\right)_{3}$ fragments (see ref. 32 and 34 and S. Kronig, E. Theuergarten, C. G. Daniliuc, P. G. Jones and M. Tamm, Angew. Chem., Int. Ed., 2012, 51, 3240).

57 W. Kohn, A. D. Becke and R. G. Parr, J. Phys. Chem., 1996, 100, 12974.

58 (a) A. D. Becke, Phys. Rev. A, 1988, 38, 3098; (b) C. T. Lee, W. T. Yang and R. G. Parr, Phys. Rev. B: Condens. Matter Mater. Phys., 1998, 37, 785.

59 (a) A. D. McLean and G. S. Chandler, J. Chem. Phys., 1980, 72, 5639; (b) R. Krishnan, J. S. Binkley, R. Seeger and J. A. Pople, J. Chem. Phys., 1980, 72, 650.

60 E. D. Glendening, A. E. Reed, J. E. Carpenter and F. Weinhold, NBO Version 3.1.

61 (a) T. Guo, S. Dechert and F. Meyer, Organometallics, 2014, 33, 5145; (b) M. Heckenroth, A. Neels, M. G. Garnier, P. Aebi, A. W. Ehlers and M. Albrecht, Chem.-Eur. J., 2009, 15, 9375.

62 Under these conditions the maximum conversion observed of 1 to 5 was $75 \%$. Extended reaction times ( $48 \mathrm{~h}$ ) did not lead to an increase in this conversion.

63 A. R. Kennedy, J. Klett, R. E. Mulvey and S. D. Robertson, Eur. J. Inorg. Chem., 2011, 4675.

$64 \mathrm{ZnR}_{2} \cdot \mathrm{IPr}$ was prepared by mixing equimolar amounts of $\mathrm{ZnR}_{2}$ and IPr in hexane. See ESI for experimental details and X-ray crystallographic and spectroscopic characterisation.

65 H. Clavier and S. P. Nolan, Chem. Commun., 2010, 46, 841861. 
66 The relative stabilities of IPr/aIPr have previously been computed using a different level of theory (ZORA-BLYP/ TZ2P level using COSMO to simulate the solvent effect), with $\Delta E_{\mathrm{a}-\mathrm{n}}=12.5 \mathrm{kcal} \mathrm{mol}^{-1}$, see ref. 30 .

67 As shown in Table S4 $\$$ and in agreement with the previous calculations for IPr and IMes, normal free $I^{t} \mathrm{Bu}$ is $17.2 \mathrm{kcal}$ $\mathrm{mol}^{-1}$ more stable than its free abnormal isomer.

68 R. M. Stolley, H. A. Duong, D. R. Thomas and J. Louie, J. Am. Chem. Soc., 2012, 134, 15154.

69 Attempts to study transformation of $\mathbf{1}$ into 5 under pseudofirst-order conditions (using a 10 molar equivalent excess of $\mathrm{GaR}_{3}$ per complex 1) completely inhibited the isomerization process.

70 R. B. Jordan, Reaction Mechanisms of Inorganic and Organometallic Systems, Oxford University Press, Oxford, UK, 3rd edn, 2007.

71 Consistent with this interpretation, the formation of $\mathrm{aI}^{t} \mathrm{BuGaR}_{3}$ (8) from an equimolar mixture of $\mathrm{I}^{t} \mathrm{Bu}$ and $\mathrm{GaR}_{3}$ occurs under much milder conditions (room temperature, 60 minutes) than for 5 . In this case $\mathrm{I}^{t} \mathrm{Bu}$ and $\mathrm{GaR}_{3}$ fail to form a stable normal adduct, thus a dissociation step is not required.

$72 \mathrm{NMR}$ studies of equimolar amounts of $\operatorname{IPr}^{\mathrm{D}} \cdot \mathrm{GaR}_{3}\left(\mathbf{1}^{\mathrm{D}}\right)$ and $\mathrm{IPr} \cdot \mathrm{GaR}_{3}(\mathbf{1})$ in $d_{8}$-THF were carried out in order to assess if the transformation of $\mathbf{1}$ to $\mathbf{5}$ occurs via intra- or intermolecular mechanism. However analysis of the NMR data proved to be inconclusive due to the overlapping of the signals from the protonated and deuterated species.

73 In particular the reactivity of $\mathbf{1}$ can be related to that described by Tamm for $\mathrm{I}^{t} \mathrm{Bu} \cdot \mathrm{B}\left(\mathrm{XyF}_{6}\right)_{3}$ that forms a stable normal adduct at room temperature but heating at $110{ }^{\circ} \mathrm{C}$ isomerizes to $\mathrm{aI}^{t} \mathrm{Bu} \cdot \mathrm{B}\left(\mathrm{XyF}_{6}\right)_{3}$. This system exhibits FLP reactivity and can activate $\mathrm{CO}_{2}, \mathrm{HCCPh}$ and THF, as reported in ref. 34 .

74 T. A. Rokob, A. Hamza, A. Stirling and I. Pápai, J. Am. Chem. Soc., 2009, 131, 2029. 